Werner Bauer, Präsident des SIWF, unterzieht das heikle Gleichgewicht zwischen Reglementierung und Eigenverantwortung, zwischen Freiheit und Sicherheit nach- stehend einer scharfsinnigen und sehr treffenden Analyse. Für seinen Beitrag sei ihm herzlich gedankt! Dr. med. Jacques de Haller, Präsident der FMH

\title{
Ärztliche Bildung - akkreditiert, reglementiert, evaluiert, finanziert und optimiert?
}

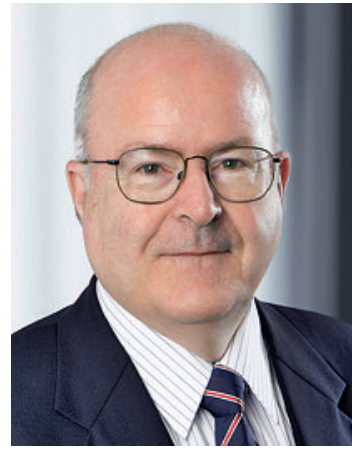

Zweifellos besteht ein grosses öffentliches Interesse an gut aus- und weitergebildeten Ärztinnen und Ärzten, die während des ganzen Berufslebens ihre Kenntnisse auf dem aktuellen Stand halten.

Die ärztliche Weiter- und Fortbildung bewegt sich deshalb in einem Spannungsfeld zwischen Reglementierung, Aufsicht und Selbstverantwortung. Für das Schweizerische Institut für ärztliche Weiter- und Fortbildung SIWF und für die Fachgesellschaften bedeutet dies, die Qualität der Bildung glaubhaft sicherzustellen und dabei so viel Freiheit als möglich zuzulassen. Das ist nicht immer einfach: Sind Reglementierungen und Kontrol-

\section{Die Qualität sicherzustellen und so viel Freiheit als möglich zuzulassen - in diesem Spannungsfeld steht die ärztliche Bildung.}

len im Empfinden der Ärzte zu strikt, hört man bald einmal Wörter wie «Kindergarten» oder «Alibibürokratie». Verlässt man sich mehr auf lockere Empfehlungen und eigenverantwortliches Verhalten, wird nach dem Einschreiten gegen «schwarze Schafe» und nach einer aussagekräftigen Evaluation gerufen.

Zur Fortbildung ist die Ärzteschaft zwar verpflichtet, in der Ausgestaltung ist sie frei. Die Fortbildungsordnung erlaubt es, die individuellen Bedürfnisse je nach Tätigkeitsbereich abzudecken - ganz im Sinne des «continuing professional development», wie die Fortbildung im angelsächsischen Raum heute heisst.

Ohne Anerkennungsverfahren und Crediterteilung geht es allerdings nicht, würde uns doch sonst schnell völlige Beliebigkeit und fragliche Qualität vorgeworfen. Selbstverständlich ist, dass wir uns laufend Gedanken machen, wie die Fortbildung methodisch und inhaltlich auf die Bedürfnisse der Zukunft ausgerichtet werden kann.

Die Verantwortung für die Weiterbildung liegt nicht mehr voll in ärztlichen Händen. Das SIWF als Organ der
FMH erteilt im Mandat der Eidgenossenschaft die Facharzttitel auf der Grundlage des Medizinalberufegesetzes. Dieses befindet sich gerade in Revision, und es ist klar erkennbar, dass der Trend bei den Gesetzesschreibern nicht in Richtung von mehr Freiheit, sondern in Richtung von mehr Regulierung und staatlicher Kontrolle geht. Noch sind die Paragraphen nicht in Granit gemeisselt, doch es wird Überzeugungskraft und politischen Einsatz brauchen, um die vielbeschworene «Public-private partnership» nicht zur Beziehung eines öffentlichen Seniorpartners mit einem privaten Juniorpartner degenerieren zu lassen.

Eines muss in diesem Zusammenhang gesagt sein: Der Satz «Wer zahlt, befiehlt» hat auch eine Umkehrung: Wer befiehlt, zahlt! Die Weiterbildung wird staatlich reglementiert und überwacht. Ohne ein gutstrukturiertes Weiterbildungssystem ist die Zukunft des ärztlichen Pfeilers unseres Gesundheitswesens bedroht - es geht zweifellos um ein «devoir public». Deshalb muss jetzt zeitgerecht ein Finanzierungsmodell für die Weiterbildung erarbeitet und in allen Kantonen umgesetzt werden.

Alle sieben Jahre werden die Facharzt-Weiterbildungsgänge neu «akkreditiert». Damit wird auch das staatliche Mandat für das SIWF erneuert. Dieser Prozess hat soeben stattgefunden und ist mit einer Verfügung des Eidgenössischen Departements des Innern für jeden Facharzttitel abgeschlossen worden. Die Akkreditierung stützt sich auf den Selbstbeurteilungsbericht jeder Fachgesellschaft, auf Expertenberichte und auf Visitationen. Sie ist begleitet von Empfehlungen oder von bindenden Auflagen, die bis zu einem bestimmten Termin umgesetzt werden müssen. Die Allgemeine Innere Medizin zum Beispiel hat die Auflage erhalten, eine Beurteilung am Ende der Weiterbildung einzuführen, die auch die praktischen Fähigkeiten erfasst. Eine Möglichkeit dazu bieten die arbeitsplatzbasierten Assessments. Für alle Fachgebiete wurde zudem empfohlen, elektronische Logbücher einzuführen und die Weiterbildenden mit «Teach the teachers»-Projekten gezielt zu unterstützen.

Ob nun akkreditiert, reglementiert, evaluiert oder nach finanziellen Mitteln gesucht wird - entscheidend ist, dass nicht «l'art pour l'art» betrieben wird, sondern dass alle Massnahmen im Dienste der Optimierung der ärztlichen Bildung stehen. Daran arbeitet das SIWF.

Dr. med. Werner Bauer, Präsident des Schweizerischen Instituts für ärztliche Weiter- und Fortbildung SIWF 